\title{
En guise de postface : tre svenska haiku
}

Jérôme David

Université de Genève

Citons d'emblée deux haïkus en suédois :

De sparkar fotboll

plötsligt förvirring - bollen

flög över muren

Mörker över snön -

endast vågskummet

gör havet synligt

Le premier se plie rigoureusement aux règles métriques du haïku japonais dit «traditionnel » : un tercet de 5, 7, puis 5 syllabes. Le second, tout aussi concis et ternaire, se permet sur ce point une entorse ; elle demeure cependant minime. Tous les deux évoquent une observation ou une impression située : on joue au football au printemps, en été peut-être ; la neige fixe son propre calendrier. L'exigence du « mot de saison » - le kigo —, autre trait caractéristique du haiku standard, est satisfaite. Il n'est pas jusqu'à la troisième des composantes de cette poétique minimale du haïku qui ne soit respectée. La césure interne au tercet signalée en japonais par un " mot de coupure » ou kireji intraduisible comme tel, car exempt de contenu sémantique - est en effet indiquée par un tiret. Le premier poème en joue avec une sorte d'effet de retardement ; le tiret annonce la déliaison du tercet dès le deuxième vers mais c'est un contre-rejet qui l'exécute ; la chute du troisième vers s'en détache d'autant, découplée désormais du distique précédent. Le second haïku isole le premier vers des deux autres comme le décor où va se jouer l'expérience poétique. Versification impaire, indexation sur une saison et césure interne au tercet où s'engouffre l'implicite du monde : ces deux poèmes épousent les contraintes de

How to cite this book chapter:

David, J. 20I 5. En guise de postface : tre svenska haiku. In: Cedergren, M. et Briens, S. (eds.) Médiations interculturelles entre la France et la Suède. Trajectoires et circulations de I945 à nos jours. Pp. 28 I-288. Stockholm: Stockholm University Press. DOI: http:// dx.doi.org/ıo.I6993/bad.u. License: CC-BY 
ce que l'on en est venu un peu partout, et parfois même au Japon, à considérer comme le haïku véritable.

Il serait aisé d'interpréter ces tercets à travers le filtre comparatiste traditionnel : la littérature suédoise influencée par la littérature japonaise ; une espèce de japonisme suédois se reconnaissant dans un genre poétique produit à l'autre bout de la terre ; les caractéristiques culturelles des écrivains suédois achevant de donner à cette réception une touche particulière. Mais cette interprétation devrait répondre à une série de questions redoutables qui, à tout le moins aujourd'hui, ne trouvent plus de réponses aussi évidentes que par le passé : quelle conception de la nationalité littéraire nous autoriserait à opposer ainsi, en blocs détachés de tout contexte, la Suède et le Japon ? Tous les écrivains "suédois » partagent-ils des traits communs qu'exprimeraient leurs œuvres respectives ? Que signifie « se reconnaître » dans une autre culture - et n'en existerait-il donc qu'une seule variante ? Autrement dit, nos deux poèmes sont-ils suédois indépendamment de cette référence à un genre poétique japonais ? Peut-on isoler ces formes brèves de la littérature suédoise de modèles importés d'ailleurs (d'autres pays, d'autres langues) ? Cet ailleurs est-il, en l'occurrence, exclusivement japonais ? Et le rapport à cet ailleurs est-il identique dans l'un et l'autre poèmes?

Deux partis pris s'excluent ici, que l'on pourrait résumer en deux mots d'ordre contraires : "d'abord les frontières, ensuite les échanges »; "d'abord les échanges, ensuite les frontières ». Ou, pour parler comme l'anthropologue James Clifford, les roots d'un côté, et les routes de l'autre (Clifford, I997). Bref, l'Etat-nation, la modernité, la citoyenneté exclusive, d'un côté ; de l'autre, les communautés aux territoires pluriels, la longue durée historique (du Moyen-Âge au XXI ${ }^{e}$ siècle) où la nation est presque une exception, le cosmopolitisme.

La Suède tire-t-elle son existence d'une suéditude essentielle, qu'elle aurait inégalement exprimée au fil des siècles, ou d'un lent travail collectif de stabilisation géographique, politique et moral d'une communauté toujours précaire de sujets, de citoyens et d'habitants ? La littérature comparée, lorsqu'elle postule la différence constitutive des cultures qu'elle met en miroir, ne fait que reconduire un imaginaire de l'appartenance qui, pour l'essentiel, ne correspond plus à nos expériences ordinaires. Et, dans le cas du haïku, l'éloignement spatial, voire culturel, de la Suède et du Japon ne change rien à cet axiome de l'analyse : sachant l'importance de la littérature et de l'histoire littéraire dans la légitimation des Etats-nation aux $\mathrm{XIX}^{\mathrm{e}}$ et $\mathrm{XX}^{\mathrm{e}}$ siècles, les études littéraires 
gagneraient à interroger leurs objets - la littérature "suédoise ", la littérature "japonaise » —à l'horizon des reterritorialisations à l'œuvre aujourd'hui à l'échelle des mégapoles, des régions ou des continents.

\section{Tokyo-London-Paris-Stockholm}

Le premier haiku est tiré du recueil Fängelse de Tomas Tranströmer, paru en I959. Le deuxième a gagné le concours annuel de la Swedish Haiku Society en 2003 ; son auteur s'appelle Tore Sverredal. L'un a obtenu le prix Nobel ; l'autre publie sur Facebook. Ce qui différencie toutefois le plus ces deux poèmes, ce n'est pas la légitimité très inégale de leur auteur, mais l'époque de leur rédaction. Publier un haïku en Suède à la fin des années I 950 n'avait pas les mêmes implications qu'au début du XXI $\mathrm{XI}^{\mathrm{e}}$ siècle. Ou plutôt, opter pour la forme du haïku dans les années I950-I960 n'engageait pas le même rapport à la poésie, à la langue, au Japon et aux échanges culturels qu'aujourd'hui. La route empruntée par le haïku pour parvenir en Suède ne fut pas identique. Et dès lors que l'on accole des noms propres aux poèmes, comme je viens de le faire, cette histoire devient cruciale.

Le recueil de Tranströmer découle d'une fascination pour la culture asiatique que partageaient dans ces années-là, en Suède, aussi bien Harry Martinson que Bo Setterlind ou Dag Hammarskjöld. Dans le cas de Tranströmer, cependant, et peut-être pour les trois autres également, la relation à la poésie japonaise fut largement médiatisée par la redécouverte, en France, du haïku dans les années I950-1960.

René Char, Paul Éluard et Guillevic seraient les marques de ce détour. Ils figurent au sommaire de I9 moderna franska poeter, édité par Erik Lindegren et Ilmar Laaban (1948), dont la femme de Tranströmer a pu dire dans Le Monde du 5 janvier 20I 2 qu'il s'agissait à l'époque du livre de chevet de son mari. C'est l'indice, sinon la preuve, d'une intercession française dans sa trajectoire d'écrivain en faveur des formes poétiques très brèves et, dans leur sillage, du haïku.

À Paris, en effet, l'intérêt pour cette forme poétique regagna aux alentours de 1950 une vigueur qu'elle avait perdue depuis la fin des années I920. Après une première effervescence autour de ce que l'on appelait encore le haï-kaï durant le premier tiers du $\mathrm{XX}^{\mathrm{e}}$ siècle - effervescence qui réunit une communauté hétéroclite de haijins francophones comme Paul-Louis Couchoud, Julien Vocance, Jean-Richard Bloch ou, pour en citer les plus connus aujourd'hui, Paul Eluard et Jean Paulhan -, une deuxième période s'ouvrit en effet à partir des années I950. Une antho- 
logie de haïkus en anglais, éditée par Reginald H. Blyth entre I949 et I952, fut l'un des déclencheurs de cet engouement renouvelé, rejouant ainsi l'effet qu'avait produit un demi-siècle plus tôt, sur Paul-Louis Couchoud surtout, une autre anthologie en anglais éditée par Basil Hall Chamberlain (I 880).

Durant les années I900-I920, le haïku avait été associé à l'expression rigoureuse des sensations élémentaires. Le poème japonais rejoignait, disait-on, les efforts menés en France pour évoquer la ténuité et la fugacité de certaines expériences. Bashô était jugé compatible avec Mallarmé et Verlaine : "l'absente de tout bouquet » avait sa contrepartie dans le bouddhisme zen et "l'Impair / Plus vague et plus soluble dans l'air " s'accommodait sans heurt de la versification en cinq et sept syllabes. Le haïku était entré dans la ronde des expérimentations poétiques. L'émergence d'une " poésie objective » dans les années I940, combinée au souci de la forme brève, chez Guillevic par exemple, prolongea ces accointances de la « disparition élocutoire du poète » héritière de Mallarmé avec la contemplation détachée du haijin.

À partir des années I950, l'attention pour le haïku répondit à des préoccupations similaires : n'y aurait-il pas dans cette forme japonaise à la simplicité inégalée, avançait-on, une sorte de résorption du langage dans le langage qui ouvrirait, précisément, un accès plus direct au réel ? L'image ne s'y abolit-elle pas pour appeler à une expérience infralangagière du monde ? L'ineffable n'y trouve-t-il pas un lieu où se laisser deviner? Ce fut la position de Philippe Jaccottet. La différence avec les premières décennies du siècle tenait désormais à l'abandon de tout débat, ou presque, sur la versification. L'affaire semblait entendue. Le vers était devenu souple, structuré par son propre rythme intérieur, et les règles trop rigides en la matière ressortissaient à l'âge obsolète de la rhétorique. Le haiku n'était plus un prétexte à controverse versificatoire, comme il avait pu l'être trente ans auparavant (faut-il s'en tenir strictement aux dix-sept syllabes ? la rime en est-elle exclue ? etc.). Il exemplifiait dorénavant, par sa césure interne (kireji), une forme d'expérience esthétique fondée sur le suspens du langage à l'orée d'un réel inexprimable comme tel.

Au lieu de s'attacher à la lettre formelle du haïku, Philippe Jaccottet ou Yves Bonnefoy associèrent cette forme à une méditation sur le langage et à une métaphysique du signe : la "poésie de la présence ", comme on a parfois qualifié leurs œuvres, se découvrait dans la tradition japonaise un alter ego esthétique. Plus encore, le haïku dessinait à leurs yeux la levée de certaines des apories sur lesquelles butaient leurs 
efforts d'écriture dans les années I950. Jaccottet poursuivit ensuite sa recherche du dénuement (moins d'éloquence, moins d'image, moins de poète et de "poésie » dans les poèmes). Bonnefoy triangula sa propre relation à Mallarmé en y ajoutant un troisième terme éloigné, si bien que la poésie japonaise devint pour lui une ressource précieuse pour penser certaines impasses du projet mallarméen (la sortie du langage par les Idées, plutôt que par l'ordinaire et le commun).

\section{Si les formes voyagent, elles ne s'arrêtent pas moins de temps en temps}

Ces deux implantations successives du haïku en France, préparées chaque fois par une anthologie en anglais, nous renseignent sur les processus à l'œuvre lors d'échanges littéraires entre cultures éloignées. Tout d'abord, une forme importée doit s'inscrire dans des débats littéraires locaux (nationaux, régionaux) pour y devenir pertinente. L'esthétique de la suggestion poétique et le refus de l'éloquence, qui perdura en France durant les deux premiers tiers du $\mathrm{XX}^{\mathrm{e}}$ siècle, étaient propices à l'accueil et à la relance du haïku.

Mais cette pertinence de la forme importée tient aussi à son contexte initial d'émergence ; ou, plus précisément, aux relations qu'entretiennent les cultures littéraires source et cible : l'admiration, la concurrence et la haine (et la guerre, paradoxalement) sont favorables aux emprunts, tandis que l'indifférence ou l'ignorance les rend improbables, sinon impossibles. Le haiku a ainsi circulé vers la France au début du $\mathrm{XX}^{\mathrm{e}}$ siècle parce qu'il était orienté vers l'Europe. On doit en effet au

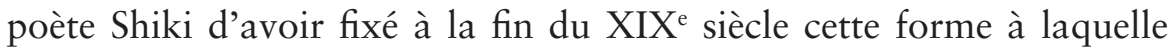
ses variations antérieures (tanka, renga, senry $\hat{u}$, etc.) refusaient depuis près d'un millénaire son autonomie de tercet : le haïku proprement dit naquit alors du détachement des trois premiers vers jusque-là agencés dans des formes plus longues, voire sérielles, et de l'exercice de sa pratique hors de toute prose (par opposition aux journaux de pèlerinage de Bashô, où un haïku résumait ou rehaussait souvent un moment particulier dans une expérience par ailleurs racontée au fil de la plume). Or Shiki développa ses réflexions sur le haïku en réaction à l'importation massive de romans anglais, russes et français au Japon sous l'ère Meiji. Le haïku, envisagé de la sorte, répondait à la nationalisation des littératures européennes - par l'invention d'une tradition poétique « japonaise » susceptible de témoigner à son tour en faveur de la grandeur culturelle pluriséculaire du pays. Désormais associé à une littérature 
nationale, le haïku avait un passeport ; il pouvait être reçu dans les autres littératures nationales.

Les poètes français ne dialoguèrent donc pas avec les Japonais en vase clos. Les intellectuels patriotes japonais de la fin du XIX ${ }^{\mathrm{e}}$ siècle se réinventèrent une nationalité littéraire au contact des romanciers européens ; les Parisiens découvrirent le haïku dans des anthologies en anglais ; ils y reconnurent le Japon, c'est-à-dire l'idée qu'ils se faisaient de la culture nationale de cet archipel. Ce qui fut le cas pour la France le fut également pour la Suède. Les échanges culturels entre deux nations seulement sont l'exception. Et si l'on problématise un tant soit peu les processus de nationalisation des littératures intervenus massivement aux $\mathrm{XIX}^{\mathrm{e}}$ et $\mathrm{XX}^{\mathrm{e}}$ siècles, il n'en existe pas.

\section{Les trois mondialisations du haïku}

Ce qui attisa donc en Suède l'intérêt de Tranströmer pour le haïku, ce fut sans doute une forme japonaise traduite en anglais et discutée, sinon pratiquée, par des poètes français. Plus largement, les Français autant que les Suédois furent alors les acteurs d'un engouement mondial pour le haïku. Imma von Bodmershof, par exemple, publiait à la même époque en allemand son recueil Haiku (I962), et Jack Kerouac ses Books of Haiku (I968).

Les années I950-I980 correspondirent à la seconde circulation mondiale du haïku. Les poètes français des années I900-I920 contribuèrent en effet à la première mondialisation de cette forme poétique aux côtés d'Ezra Pound, Giuseppe Ungaretti, José Juan Tablada ou Rabindranath Tagore. Entre les deux vagues, dans les années I930I940, survint une étrange amnésie collective : le genre dépérit ; et on en fit la redécouverte ébahie dès les années I950. À ma connaissance, cette énigme de l'histoire littéraire n'a pas encore été élucidée.

Tore Sverredal participe d'une troisième mondialisation du haiku. Depuis les années I980, en effet, cette forme fait partie dans le monde entier - et non plus seulement au Japon - de la gamme des pratiques ordinaires de la littérature. On ne compte plus les haijins amateurs : ils publient leurs poèmes à compte d'auteur pour eux et leurs proches; ils en ponctuent leurs discours officiels, à l'image de l'ancien président du Conseil Européen Herman von Rompuy ; et il leur arrive de fonder des associations régionales, nationales ou internationales de haïku.

Ces associations ont fleuri sur tous les points du globe: Haiku Society of America (I968); Deutsche Haiku Gesellschaft (1988) ; International 
Haiku Association (1989) ; British Haiku Society (1990) ; Svenska Haiku Sällskapet (1999); World Haiku Association (2000); Australian Haiku Association (2000); Association francophone de haïku (2003); Irish Haiku Society (2006); Association pour la promotion du haïku (2007) ; etc. Elles sont le prolongement faiblement institutionnalisé de sociabilités entre dilettantes liés par une passion commune pour le haïku. Ces activités associatives donnent le plus souvent lieu à l'édition de bulletins, à la création de sites internet plus ou moins éphémères, à des concours annuels et à des lectures publiques. Leur territoire d'action variable - un pays, une langue, un continent, le monde - n'entraîne aucune hiérarchie particulière. Ce tissu n'est pas coordonné en réseau doté d'instances faîtières. Il s'agit avant tout de groupes disparates d'individus qui se font plaisir et le font savoir.

En d'autres termes, le XXI e siècle a hissé le haïku au sommet des genres poétiques les plus populaires de la planète. Le tercet contemplatif d'environ dix-sept syllabes est la forme qu'adoptent spontanément les littérateurs du monde entier. Je sais que mes enfants choisiront ce format pour dévider leur premier chagrin d'amour, au lieu du sonnet baudelairien qui me consolait jadis. C’est désormais le modèle par défaut de l'expression poétique.

Le succès unanime de cette forme déplace la question de sa littérarité. Comment composer des haïkus en écrivain, plutôt qu'en amateur? En France, Antoine Volodine a répondu à cette question dans un recueil attribué à l'un de ses pseudonymes : Haïkus de guerre, de Lutz Bassmann (Verdier, 2007). Le travail d'écriture ne s'y exerce plus seulement à l'échelle de chacun des poèmes, mais également au plan de leur mise en série. Le même vers revient ainsi comme une ritournelle dans plusieurs haïkus successifs - avec pour effet, d'une part, de créer entre eux des renvois qui relativisent la singularité supposée de l'expérience associée à chaque tercet et, d'autre part, de faire dériver la simplicité de leur expression non pas tant d'une quête poétique du dénuement que de la reprise d' "éléments de langage ", pour ainsi dire, tirés comme toujours chez Volodine des langues de bois politiques du $\mathrm{XX}^{\mathrm{e}}$ siècle. Le haïku comme enveloppe creuse d'une expérience trop générale pour être personnelle ; un syllogisme de l'absurde.

Tore Sverredal, à l'évidence, se démarque de Volodine. Son poème parle d'une épiphanie hivernale face à la nature. Il cherche à fixer et à transmettre une expérience unique, mais susceptible d'être partagée. Et Sverredal ne l'a sans doute pas écrit pour tourner en dérision le marketing touristique scandinave et ses images d'Épinal. 
Et Tranströmer ? En 2004, dans La Grande énigme (Den stora gåtan), il est resté fidèle à ses partis pris de I959. Déjà hors du temps où les mouches s'épuisent en vain :

Rentjur i solgass.

Flugorna syr och syr fast

skuggan vid marken.

\section{Bibliographie}

Bassmann, L. (2007), Haïkus de guerre. Paris :Verdier.

Blyth, R. H. (I949-I952), Haiku. The Hokuseido Press (4 vol.).

Bodmershof (von), I. (r962), Haiku. München/Wien : Langen-Müller.

Chamberlain, B. H. (г880), The Classical Poetry of the Japanese by Basil Hall Chamberlain. London : Trübner.

- (1902), "Bashô and the Japanese poetical epigram ». Transaction. Asiatic Society of Japan 30 : 244-362.

Clifford, J. (1997), Routes. Travel and Translation in the Late Twentieth Century. Cambridge : Harvard Univ. Press.

Kerouac, J. [1968] (2003), Books of haikus. New York : Penguin Poets.

Lindegren E. \& Laaban, I. (I948), I9 moderna franska poeter. Stockholm : Bonnier.

Svenska Haiku Sällskapet (2004), Haiku 7.

Tranströmer, T. (200I) [1959], Fängelse. Nio Haikudikter från Hällby Ungdomsfängelse. Uppsala : Edda.

- (2004), La Grande énigme (Den stora gåtan). Talence : Castor Astral. 